\title{
TEMIPERATURE AND DOPPLER COEFFICIENTS OF VARIOUS SPACE NUCLEAR REACTORS
}

\author{
Said F. Mughabghab, Hans Ludewig, and Eldon Schmidt \\ Brookhaven National Laboratory \\ Department of Advanced Technology \\ Bldg. 130 \\ Upton, New York 11973
}

(510) 282-5085

\begin{abstract}
Alsstract
Temperature and Doppler feedback effects for a Particle Bed Reactor (PBR) designed to uperate as a propulsion reatctur are investigated.

Several moderator types and compositions fuel enrichments and reactor sizes are considered in this study. From this study it could be concluded that a PBR can be contigured which has a negittive prompt feedback, zero coolant worth, and a small positive to zero moderator worth. This reactor would put the lowest demands on the control system.
\end{abstract}

\section{INTRODUCTION}

The design of Nuclear Thermal Propulsion (NTP) reactors has as one of its goals the maximization of the thrust/weight ratio. This goal can only be achieved by making the reactor core as light as possible, within the constraints of a prudent mechanical and safe design. In a Particle Bed reactor (PBR) the moderator occupies approximately $50 \%-75 \%$ of the core volume. Thus, in order to minimize the core mass, it is important to reduce the moderator mass to its lowest possible value. In view of this requirement it is desirable to use the lowest density moderator, consistent with the moderating performance required. In addition the uranium enrichment plays a role in reducing the size and thus the reactor mass. Generally, the highest enrichment corresponds to thefsmallest possible reactors. However. this reduces the fertile traction in the fuel and thus impacts the Doppler feedback.

Based on the general guide lines outlined above. it was decided to consider the following moderitor types:

1. Al/Pe - This moderator consists of aluminum foam with polyethylene. The aluminum is added to enhance the heat transter. This moderator is limited to comparativeiy low operating temperatures, and the aluminum acts as a diluent, since it is a relatively poor inoderator.

2. Be/Pe - This inoderator is similar to the one described in 1, except it uses beryllium insteald of aluminum. Its primary advantage is that beryllium aids in the moderation process. In addition, this moderator could potentially be lighter than the aluminum hassd moderator, since heryllium is a lower density material.

1
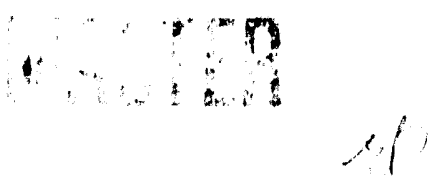
3. Be/Li-7/H - This moderator is similar to the one described in 2, with the polyethylene replaced by lithium-7 hydride. This moderator allows for a higher uperating temperature, without compromising the moderating performance of the moderator. It should also be lighter than the moderators described in 1 and 2 , for the same moderating power.

The Doppler effect in PBR's is due to a combination of resonance broadening in the fertile matterial (U238) and the hot frit coating material (Ta). Since the resonance integral for tantalum is much larger than for uranium-238, a thin coating on the hot frit has a significant effect. The uranium enrichment can still be varied to enhance the Doppler effect, ensuring a negative prompt coefficient.

\section{Reactor Description}

A brief description of a PBR will be given. More detailed descriptions are given in other papers presented at this meeting. The reactor consists of particles (.500 microns OD) contained between two co-axial porous tubes (frits). These assemblies are referred to as a tuel elements, and are embedded in moderating material. The fuel elements are arranged in a hexagonal pattern within the moderator. Coolant (hydrogen) flows into the core from one end, cooling the moderator tirst and then flows radially through the tuel element, and out the core through the duct formed by the inner frit (hot frit). The core is contained in a pressure vessel, and has inlet and outlet plena attached at the ends. An exhaust nozzle is integrated into the outlet plenum.

The reactors to be discussed in this study will consider all of the above mentioned moderators and a variety of uranium enrichments. Both 37 and 61 element reactors will be considered. Finally, two thrust levels will be considered: the first consistent with $1000 \mathrm{MW}$, and the second consistent with $2000 \mathrm{MW}$. The overall reactor geometric parameters are given below:

$\begin{array}{lccc}\text { Reactor Power } & 1000 \mathrm{MW} & 2000 \mathrm{MW} \\ \text { Case } & 1 & 2 & 3 \\ \text { Number uf Elements } & 37 & 37 & 61 ! \\ \text { Fuel Bed Volume (liters) } & 25 & 50 & 50\end{array}$

The method of analysis used in this paper is hased on the Monte Carlo method. This method was deemed necessary since PBR's are characterized by high leakage and are very heterogeneous. In addition it is important to conserve hoth volume of material and neutron leakage area. This reguirement cannot he simultaneously satistied using a method which does not model all the components explicitly. The MCNP code was used. which is based on a combinatorial geometric representation and a comtinuous energy cross section description. This makes it unnecessary to create approximate geometric representations of the core and special position dependent group cross sections.

\section{$\underline{\text { Results. }}$}

In this section the feedbatck elfects due to the change in the moderatur temperature, and thus changes in its scaltering kernel will be discussed. In addition the Doppler feedbatk and conblant worth an a function of moderator volume will be outlined. 


\section{Moderator Worth}

The variation of moderator worth with temperature has been presented in detail in a previous publication (Mughabghab, 1993). Here we will discuss the dependence of the feedback on moderator volume. Table I shows the dependence of a moderator composed of beryllium/hydrogenous material on moderator volume for Case 1.

TABLE 1. Dependence of Moderator Worth hy Component on Volume.

\begin{tabular}{|c|c|c|c|c|c|c|}
\hline \multicolumn{7}{|c|}{ (Worth in cents/K) } \\
\hline & \multicolumn{3}{|c|}{ Beryllium } & \multicolumn{3}{|c|}{ Hyúrogenous Material } \\
\hline Volume (cc) & $150(\mathrm{~K})$ & $250(\mathrm{~K})$ & $350(\mathrm{~K})$ & $150(\mathrm{~K})$ & $250\left(K^{\prime}\right)$ & $350(K)$ \\
\hline $2.36(5)^{* *}$ & -0.06 & 0.08 & 0.5 & 2.05 & 1.04 & 1.08 \\
\hline $2.86(5)$ & 0.15 & 0.49 & 0.87 & 2.89 & 1.75 & 1.35 \\
\hline $6.89(5)$ & 0.87 & 1.28 & 3.79 & 0.69 & 3.74 & 3.14 \\
\hline \multicolumn{7}{|c|}{$: 2.36(5)=2.36 \times 10^{5}$} \\
\hline
\end{tabular}

These results show that the component of worth due to heryllium increases with increasing moderator volume, and beryllium temperature. The increase with moderator volume is approximately proportional to $\mathrm{V}^{\mathrm{n}}$, where $\mathrm{n}=1.6$. In the case of the hydrogenous component, the worth also increases with increasing moderator volume, but it decreases with increasing hydrogenous material temperature. Thus it is important to determine the component temperatures when determining the moderator worth for any given average temperature. In general the metal component will respond faster (due to better heat conduction) to changes in coolant conditions, particularly during start-up transients. The hydrogenous component (hydride or polyethylene) will not respond as rapidly and thus is expected to operate at higher temperatures during start-up transient conditions in which the moderator cooling depends on both conduction and convection. Finally, it is clear that the design details of the moderator configuration are important, since long conduction paths in the beryllium will lete to a more responsive moderator temperature than long paths in the hydrogenous material.

\section{Doppler Worth}

The Doppler worth has been determined for several cores of the larger reador (Calses 2 and 3). Tahle 2 shows the variation of Doppler worth with number of elements and moderator volume. 
TABLE 2. Doppler Worth Variation with Number of Elements and Moderator Volume.
Volume (ec)
37 Elements
(Worth in Delta $k$ )
Volume (cc)
61 Elements

$\begin{array}{llll}2.40(5) & -0.026 & 2.52(5) & -0.024 \\ 4.02(5) & -0.009 & 4.27(5) & -0.015 \\ 6.12(5) & -0.016 & 0.50(5) & -0.011\end{array}$

The above results show that the Doppler worth drops off with increasing moderator volume. This etfect is due to the softening of the neutron spectrum and the lower fraction of neutrons in the resonance energy band. The difference between the (wo reactor contigurations is small, despite the thicker fuel beds, and thus larger disadvantage tactor in the case of the 37 element core.

\section{Hydrogen Worth}

Hydrogen is the coolant used in propulsion reactors, and thus the effect of introducing such a good moderator into the core needs to be studied, particularly for start-up transients. The magnitude of the hydrogen worth depends on whether the core is over- or under-moderated. Furthermore, the moderator volume determines whether a core is over- or under-moderated. Thus, there is a strong correlation between the hydrogen worth of a core and its moderator volume. Table 3 shows the hydrogen worth for Case 2.

TABLE 3. Hydrogen Worth as a Function of Moderator Volume.

Moderator Volume (ec)

Hydrogen Worth (deltak)

$\begin{array}{ll}2 .+(1)(5) & +0.029 \\ +.02(5) & -0.011 \\ 0.12(5) & -0.027\end{array}$

The results show that the hydrogen worth is a monotonicilly reducing function of moderator volume. The volume at which the introduction ot hydrogen hats zero worth, generally corresponds to a core which is neither under- or wer-molerated, and would thus pose the smallest enntrol regurements.

\section{Conclusions}

The bollowing condusions can be drawn from this study: 
1. The moderator leedback due to scattering kernel effects is always positfve, although it becomes exceedingly small for some designs, and may he over-rodden in certain temperature ranges by the other effects i.e. expansion and dissociation (hychrides).

2. In the calse where the moderator is composed of metal/hydrogenous material combination. their individual temperature responses needs to be determined in order to estimate the overall feedback effect. This is necessary since the metal and hydrogenous material teedhack worths vary in opposing directions.

3. For a given tuel volume, the magnitude of the Doppler effect is not sensitive to the number of clements.

4. The magnitude of the Doppler etfect decreases with increasing moderator volume.

5. A moderator size cun be detined at which the introduction of hydrogen conlan has no etfect on the multiplication factor. This implies a neutrally moderated system.

0. A PBR can be contigured which has a negative prompt teedhack costlicient, zero condant worth, and at small to zero moderator worth. This realctor would put the lowest demands on the control system.

\section{Acknowledgements}

Research carried out under the auspices of the U.S. Department of Energy under contract No. DEAC02-76CH0OO16.

\section{$\underline{\text { References }}$}

1. Mughabghab.S.. H. Ludewig. E. Schmidt.(1993) Tenth Symposium on Space Nuclear Power and Propulsion, Jan 10-14(1993) Alhuquerque. NM, D. 965, editors M. El-Genk and M.D. Howver (AIP Cunterence Proceedings 271). 
